\title{
Le rat RICO (génétiquement hypercholestérolémique) : un bon modèle pour tester un composé alimentaire ou une drogue spécifique d'une enzyme-clé du métabolisme du cholestérol ?
}

\author{
Claude Lutton \\ Laboratoire de physiologie de la nutrition, unité associée, université Paris-Sud-XI/Inra, \\ bâtiment 447, université Paris-Sud, 91405 Orsay cedex, France
}

(Reçu le 7 décembre 1998 ; accepté le 16 mai 1999)

\begin{abstract}
The genetically hypercholesterolemic RICO rat: a good model for testing a food substance or a drug specific for a key enzyme involved in cholesterol metabolism? The genetically hypercholesterolemic RICO rat, whose cholesterolemia is situated between 1.3 and $1.5 \mathrm{mg} \cdot \mathrm{mL}^{-1}$, possibly reaching $2 \mathrm{mg} \cdot \mathrm{mL}^{-1}$, after the addition of cholesterol to its food, possesses a different lipoprotein spectrum than man, because approximatively $70 \%$ of the plasma cholesterol is carried by HDL ( $28 \%$ of which are carried by the light HDLl subfraction, rich in apolipoproteinE (apoE). The effects of certain substances in food (carbohydrates, cholesterol, allyldisulfide, etc.) or drugs (ethinylestradiol, streptozotocin, statins, inhibitors of ACAT, etc.) on the cholesterolemia of the rat were studied, in relation to certain important parameters of cholesterol metabolism (LDLr, VLDL liver secretion, activities of lipolytic enzymes: LPL, HL, etc.). The increase in a number of LDL receptors (LDLr) in the RICO rat, induced by ethinylestradiol, streptozotocin, etc., provokes an important decrease in the apoE-rich HDL concentration, filtered out by its receptors. This decrease is observed in man for LDL. Simvastatin, which stimulates LDLr in man and not in rat, lowers the level of LDL in man and has no effect on the cholesterolemia of the RICO rat. In rat and man, the concentration of plasma cholesterol is inversely proportional to the rate of cholesterol synthesis in the organism and to its plasma turnover rate. The concentration of cholesterol in the plasma carried by the HDL1 of the rat, is however, proportional to hepatic cholesterogenesis. This fraction is positively correlated to the activity of hepatic lipase (HL) and negatively to the activity of lipoprotein lipase (LPL), released by heparin. These data demonstrate the importance of the liver and lipolytic enzymes in the intraplasmatic hydrolysis of HDL3 (precursors of HDL1), murine particles that can be considered similar to human LDL. (C) Inra/Elsevier, Paris.
\end{abstract}

LDL receptors / cholesterogenesis (hepatic, intestinal) / intestinal cholesterol absorption coefficient / ACAT inhibitors / HMGCoAR (statins)

E-mail : claude.lutton@ibaic.u-psud.fr 
Résumé - Le rat génétiquement hypercholestérolémique RICO, dont la cholestérolémie est comprise entre 1,3 et $1,5 \mathrm{mg} \cdot \mathrm{mL}^{-1}$, mais qui peut atteindre $2 \mathrm{mg} \cdot \mathrm{mL}^{-1}$ après addition de cholestérol à l'alimentation, possède un spectre lipoprotéinique différent de celui de l'homme puisque $70 \%$ du cholestérol du plasma est transporté par les HDL dont une bonne part ( $28 \%)$ par des HDL1 légères, riches en apoE. Les effets de plusieurs composés de l'alimentation (carbohydrates, cholestérol, allyldisulfide, etc.) ou de diverses drogues (éthinylestradiol, streptozotocine, statines, inhibiteurs de l'ACAT, etc.) ont été étudiés sur la cholestérolémie de cet animal, en relation avec certains paramètres importants du métabolisme du cholestérol (LDLr, sécrétion des VLDL par le foie, activités des enzymes lipolytiques : LPL, HL, etc.). L'augmentation du nombre de récepteurs aux LDL (LDLr) chez le rat RICO induite par l'éthinylestradiol, la streptozotocine, etc., entraîne une baisse importante de la concentration des HDL1, riches en apoE, épurées par ces récepteurs ; cette baisse serait observée au niveau des LDL, chez l'homme. La sinvastatine, stimulatrice des LDLr chez l'homme et non chez le rat, abaisse la LDLémie humaine et n'a pas d'effet sur la cholestérolémie du rat RICO. Chez le rat et l'homme, la concentration plasmatique du cholestérol est inversement proportionnelle au débit de synthèse du cholestérol par l'organisme et à son taux de renouvellement plasmatique, mais la concentration du cholestérol du plasma porté par les HDL1 du rat est proportionnelle à la cholestérogenèse hépatique. De même, cette fraction est positivement corrélée à l'activité de la lipase hépatique (HL) et négativement à celle de la lipoprotéine lipase (LPL), relarguables par l'héparine. Ces données montrent l'importance du foie et des enzymes lipolytiques dans l'hydrolyse intraplasmatique des HDL3, précurseurs des HDL1, particules murines qui peuvent donc être rapprochées des LDL de l'humain. (C) Inra/Elsevier, Paris.

LDL récepteurs / cholestérogenèse (hépatique, intestinale) / coefficient d'absorption intestinale du cholestérol / inhibiteurs de l'ACAT / de l'HMGCoAR (statines)

\section{INTRODUCTION}

Le rat RICO a été sélectionné originellement dans l'unité d'élevage de Ciba-Geigy (Bâle, Suisse) à partir d'un mutant albinos de Rattus norvegicus. Après la quinzième génération, ces animaux étaient hypercholestérolémiques à $100 \%$ [37]. C'est pourquoi, ces rats ont été appelés RICO (rats with increased cholesterol). Cette hypercholestérolémie, qui est établie pour les deux sexes un jour après le sevrage, augmente progressivement entre la quatrième et la dixième semaines. La cholestérolémie totale varie entre 1,3 et $1,5 \mathrm{mg} \cdot \mathrm{mL}^{-1}$ et peut s'élever à plus de $2 \mathrm{mg} \cdot \mathrm{mL}^{-1}$ après addition de cholestérol dans l'alimentation.
Les principales particularités du métabolisme du cholestérol du rat RICO par rapport au rat hétérozygote normocholestérolémique ont été décrites dans deux revues $[27,38]$. Il s' agit principalement :

- d'une cholestérogenèse plus grande ( $25 \mathrm{mg}$ par jour contre $16 \mathrm{mg}$ chez le normocholestérolémique) due à une élévation de la synthèse de cholestérol dans l'intestin grêle et le foie ;

- d'un doublement de la sécrétion de cholestérol vers la lymphe $\left(580 \mu \mathrm{g} \cdot \mathrm{h}^{-1}\right.$ contre $330 \mu \mathrm{g} \cdot \mathrm{h}^{-1}$ ), cholestérol transporté principalement par les chylomicrons et les VLDL;

- d'une élévation de toutes les classes de lipoprotéines dans le plasma mais principa-

\footnotetext{
Abréviations : CM, chylomicrons ; VLDL, lipoprotéines de très basse densité ; LDL, lipoprotéines de basse densité ; HDL, lipoprotéines de haute densité ; apoE, apolipoprotéine E ; HMGCoAR, hydroxy-méthyl-glutarylCoA réductase ; ACAT, acylCoA-cholestérol-acyl transférase; CETP, cholesteryl ester transfer protein ; LPL, lipoprotéine lipase ; HL, lipase hépatique ; LDLr, récepteur aux $\mathrm{LDL}=$ apoB $100 / \mathrm{E}$ récepteur; $\mathrm{FCR}$, fractional catabolic rate, taux de renouvellement.
} 
lement des fractions comprises entre les densités 1,020 et 1,13 . Une élévation plus forte des chylomicrons est également observée lorsque du cholestérol est ajouté à l'alimentation.

De plus, si l'activité de la lipase hépatique a été trouvée semblable chez le rat RICO et le normocholestérolémique, l'activité de la lipoprotéine lipase est plus basse $(-35 \%)$ chez le RICO. Cette dernière observation est probablement à relier au fait que l'épuration plasmatique (en termes de cholestérol) des chylomicrons et des «LDL » $(1,020<\mathrm{d}<1,050)$ du rat RICO est ralentie par rapport au rat contrôle, hétérozygote, normocholestérolémique. Le taux d'épuration (ou FCR : fractional catabolic rate) atteint $770 \pm 60$ au lieu de $1070 \pm 70 \% \cdot \mathrm{h}^{-1}$ et $5,5 \pm 0,6$ au lieu de $7,9 \pm 0,5 \% \cdot \mathrm{h}^{-1}$ pour les chylomicrons et les LDL, respectivement. Il n'en est pas de même pour le taux d'épuration des VLDL ou des HDL $(1,09<\mathrm{d}<1,21)$ qui reste inchangé chez le RICO $\left(430 \pm 60 \% \cdot h^{-1}\right.$ et $8,9 \pm 0,3 \% \cdot h^{-1}$, respectivement) comparé à celui du rat normocholestérolémique $\left(510 \pm 40 \% \cdot \mathrm{h}^{-1}\right.$ et $8,8 \pm 0,5 \% \cdot \mathrm{h}^{-1}$, respectivement) [39].

Cette plus basse épuration plasmatique des chylomicrons et des LDL n'est pas liée à une quantité plus faible de LDLr, chez le rat RICO $[13,36]$. Elle résulte de la lipoprotéine elle-même et de l'interaction particule-LDLrécepteur [39]. D'ailleurs, le poids moléculaire du LDLr du rat RICO peut apparaître sensiblement plus petit (de $4 \mathrm{kDa}$ ) que celui de l'hétérozygote ou du rat Wistar, sans que l'on sache encore quelle est la modification fine de la structure du LDL-récepteur, chez le rat RICO [36].

Comparé à celui de l'hétérozygote normocholestérolémique (SW), le foie du RICO a une capacité plus réduite d'épurer les LDL du plasma (en termes de microlitres de plasma épurés de ses LDL : $-40 \%$ quand on l'exprime par unité de poids et $-66 \%$ quand elle est exprimée pour l'organe entier). Ceci suggère une réduction de la capacité de liaison des lipoprotéines légères aux LDLr chez le rat RICO, animal qui pourrait donc être un modèle intéressant de l'hypercholestérolémie humaine modérée. Les résultats expérimentaux suivants vont nous en donner quelques exemples.

\section{ANIMAUX ET MÉTHODES}

\subsection{Produits}

Ceux ajoutés à l'alimentation (cholestérol, allyl disulfide) ou injectés à l'animal (streptozotocine) sont de la plus haute pureté possible (Sigma). Les drogues ont été fournies gracieusement par les firmes : sinvastatine (Merck Sharp et Dhome, Paris, France), crilvastatine (Pan Medica, Carros, France), F 2833 (Fabre SA, Castres, France), $\beta$-cyclodextrine (Roquette, Lestrem, France), thé Tuocha (Distrifrance, Paris, France).

\subsection{Animaux et régimes}

Les rats génétiquement hypercholestérolémiques (homozygotes) RICO et les normocholestérolémiques (hétérozygotes, $\mathrm{SW}$ ) sont élevés dans notre propre animalerie. Celle-ci, à air conditionné, possède une température constante $\left(23 \pm 1^{\circ} \mathrm{C}\right)$ et des périodes alternées de lumière et d'obscurité (8-20 h/20-8 h). Après leur sevrage, vers l'âge de 3 semaines, les rats mâles reçoivent eau et une alimentation commerciale ad libitum pendant 4 semaines. Le régime semisynthétique témoin (ST) du laboratoire a pour composition : saccharose $53 \%$, caséine $23 \%$; saindoux $9,2 \%$, mélange minéral $5 \%$, lait écrémé $4 \%$, levure de bière $2,3 \%$, cellulose et mélange vitaminé $2,5 \%$, huile de noix $0,8 \%$ et cystine $0,2 \%$ [44]. Le régime semi-synthétique « amidon » $(\mathrm{SA})$ possède la même composition, le saccharose étant remplacé par l'amidon. Le dernier jour de l'expérience, les animaux sont sacrifiés à 10:00 $\pm 1 \mathrm{~h}$, par ponction aortique, après anesthésie au nembutal. Le sang, collecté sur EDTA (4\%) et monoiodoacetamide (4\%), est centrifugé à $2200 \mathrm{~g}$ pendant $20 \mathrm{~min}$ à $4{ }^{\circ} \mathrm{C}$ pour séparer le plasma et les globules rouges.

\subsection{Séparation des lipoprotéines plasmatiques}

Elle est effectuée sur 1 ou $2 \mathrm{~mL}$ de plasma, par ultracentrifugation en gradient de densité à 
$105000 \mathrm{~g}$ pendant $24 \mathrm{~h} \mathrm{[43]}$. Vingt fractions de $0,5 \mathrm{~mL}$ sont collectées et analysées quant à leur contenu en lipides (cholestérol libre et estérifié, triglycérides, phospholipides) ou en protéines [44].

\subsection{Mesure du coefficient d'absorption intestinale du cholestérol par le principe d'occupation}

Le principe et les détails de cette méthode ont été donnés [21]. Une quantité traceuse de $\left.{ }^{[4-14} \mathrm{C}\right]$ cholestérol ( $\left.37 \mathrm{kBQ}\right)$ est mélangé à $3 \mathrm{~g}$ de régime tandis qu'une seconde dose traceuse de $\left.{ }^{1,2-3} \mathrm{H}\right]$ cholestérol $(148 \mathrm{kBq})$ est ajoutée à du Tween 80 et dispersée dans du sérum physiologique. Immédiatement après que l'animal ait ingéré la dose orale de $\left[{ }^{14} \mathrm{C}\right]$ cholestérol, il reçoit la dose de $\left[{ }^{3} \mathrm{H}\right]$ cholestérol, dans la veine du pénis, sous légère anesthésie à l'éther. Des échantillons de sang sont collectés 48 et $72 \mathrm{~h}$ après, afin de déterminer la radioactivité spécifique du cholestérol plasmatique et le rapport ${ }^{14} \mathrm{C} /{ }^{3} \mathrm{H}$ du cholestérol du plasma. Le coefficient d'absorption intestinale du cholestérol (AC) est donné par : $\mathrm{AC}=(\%$ de la dose orale par $\mathrm{ml}$ de plasma $/ \%$ de la dose intraveineuse par $\mathrm{mL}$ de plasma) $\times 100$. Mesuré dans le sang ou le plasma, ce rapport reste constant $48 \mathrm{~h}$ après l'administration des isotopes [26].

\subsection{Mesure in vivo de la cholestérogenèse hépatique}

Une analyse critique de l'utilisation de l'acétate ${ }^{14} \mathrm{C}$ pour la mesure in vivo de la synthèse du cholestérol du rat a été publiée [28]. Soixantedix minutes après l'injection sous-cutanée de 3,7 à $7,4 \mathrm{Mbq}$ de $1-\left[{ }^{14} \mathrm{C}\right]$ acétate $(1,66-2,22$ $\mathrm{Gbq} \cdot \mathrm{mmole}^{-1}$ ) dissout dans $0,2 \mathrm{~mL}$ de sérum physiologique, les rats sont tués par ponction aortique sous anesthésie au pentobarbital, le foie recueilli, ses stérols extraits et leur radioactivité mesurée par des techniques décrites [21,28].

\subsection{Mesure de l'activité HL et LPL}

Les rats, nourris ad libitum, reçoivent 20 unités d'héparine $/ 100 \mathrm{~g}$ de poids (Choay, Paris, France) par voie intraveineuse (veine jugulaire). Deux minutes plus tard, $0,5 \mathrm{~mL}$ de sang sont aspirés par la même veine. Les échantillons de plasma sont stockés immédiatement à $-20^{\circ} \mathrm{C}$.
Les activités lipase hépatique (HL) et lipoprotéine lipase (LPL) sont mesurées $1-2 \mathrm{j}$ après : $5 \mathrm{~mL}$ de plasma sont incubés à $30^{\circ} \mathrm{C}$ pendant $10 \mathrm{~min}$, avec $0,2 \mathrm{~mL}$ d'une émulsion de $1,33 \mathrm{mM}-\left[{ }^{14} \mathrm{C}\right]$ trioleylglycerol $\left(106 \mathrm{dpm} \cdot \mathrm{mmole}^{-1}\right)$ et $0,1 \mathrm{mM}$-lysophosphatidylcholine, diluée dans un tampon 0,2 M-Tris/HCL, pH 9, contenant $0,4 \%$ BSA et $0,5 \mathrm{M}-\mathrm{NaCl}$. L'activité LPL est obtenue de la même manière, sans $\mathrm{NaCl}$ ajouté et avec un sérum de rat préalablement inactivé à $56{ }^{\circ} \mathrm{C}, 30 \mathrm{~min}$. Pour plus de détails, voir Sultan et al. [47].

\subsection{Analyses statistiques}

Les données sont exprimées en moyenne \pm SEM. Les différences entre les groupes sont recherchées par le test $t$ de Student ou ANOVA (Statview 512, Abacus Concepts Inc., Calabas, Ca, États-Unis).

\section{RÉSULTATS ET DISCUSSION}

La figure 1 illustre la répartition du cholestérol dans les lipoprotéines plasmatiques du rat RICO génétiquement hypercholestérolémique et de son témoin normocholestérolique (dit $\mathrm{SW}$ ), ingérant notre régime semi-synthétique riche en amidon (SA). On observe que l'élévation de la concentration plasmatique du cholestérol chez le rat RICO concerne toutes les fractions de lipoprotéines mais principalement les fractions 5 à 13 de densité comprises entre 1,019 et 1,063 , c'est à dire les LDL et les HDL légères riches en apoE (HDL1, 1,040 $<\mathrm{d}<1,063$ ).

\subsection{Effets de quelques facteurs alimentaires}

\subsubsection{Nature des glucides}

L'effet d'une alimentation (ST) à base de saccharose (au lieu d'amidon) sur les lipoprotéines du rat RICO est également illustré dans la figure 1. La hausse de la cholestérolémie totale $\left(1,66 \pm 0,02 \mathrm{mg} \cdot \mathrm{mL}^{-1}\right.$, $n=43$, contre $1,33 \pm 0,04, n=8$, chez le těmoin ingérant le régime à base d'amidon, 
Figure 1. Distribution du cholestérol $\left(\mathrm{mg} \cdot \mathrm{mL}^{-1} \mathrm{de}\right.$ plasma) dans les lipoprotéines séparées par gradient de densité chez le rat mâle génétiquement hypercholestérolémique RICO, et le normocholestérolémique SW ingérant un régime semi-synthétique riche en amidon (SA), ou, en pointillé, chez le rat RICO ingérant le régime semi-synthétique témoin (ST), riche en saccharose. $\square$, RICO (ST) : moyenne $(n=43) ; O$, RICO (SA) : moyenne \pm SEM $(n=8) ; \diamond$, SW (SA) : moyenne $\pm \operatorname{SEM}(n=4)$.

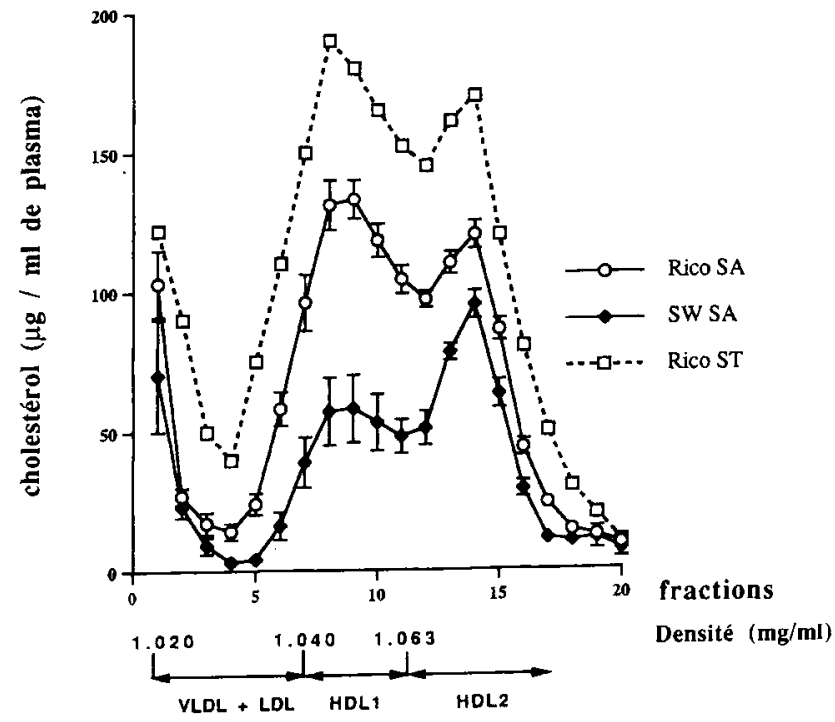

une hypercholestérolémie plasmatique plus ou moins élevée et qui touche différemment les fractions de lipoprotéines légères ou lourdes.

Chez le rat Wistar, SW ou RICO, la concentration plasmatique des chylomicrons et VLDL est toujours décuplée sous l'effet d'une alimentation riche en cholestérol $(0,5 \%)$, tandis que les fractions LDL $(1,019$ $<\mathrm{d}<1,040)$ et HDL $(1,040<\mathrm{d}<1,21)$ sont généralement sensiblement abaissées (figure 2). Pour les LDL et HDL, ces variations reflètent essentiellement une modification du nombre de particules. Pour les VLDL, en revanche, l'augmentation du cholestérol transporté est autant la conséquence de l'enrichissement en cholestérol de ces particules que d'un accroissement du nombre de particules elles-mêmes [5]. Chez le rat (Wistar et RICO), notons que le nombre de LDLr hépatiques n'est pas abaissé après addition de cholestérol à l'alimentation (observations non publiées du laboratoire) ou même a été trouvé augmenté chez le jeune rat de certaines souches [41].

Chez l'homme, dans ces mêmes conditions d'apport de cholestérol alimentaire, ce tion induit, en fonction des espèces animales, 

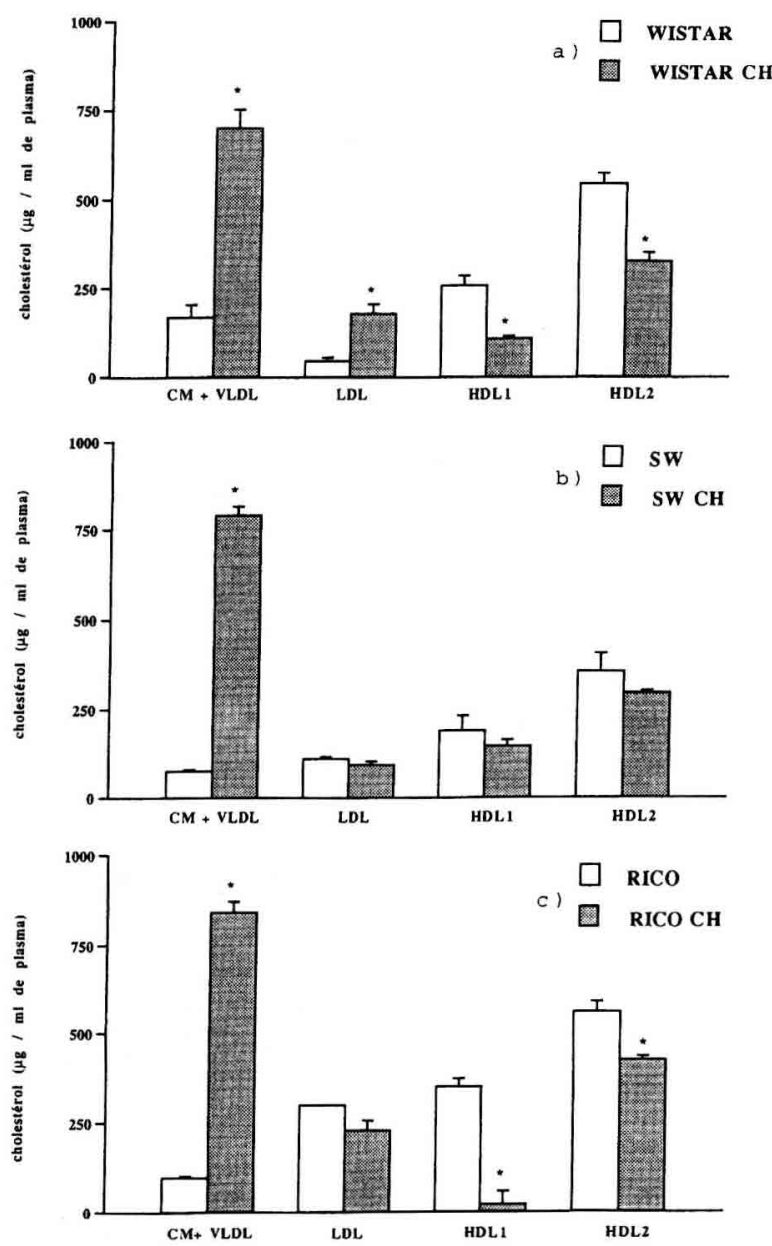

Figure 2. Distribution du cholestérol $\left(\mu \mathrm{g} \cdot \mathrm{mL}^{-1}\right.$ de plasma) dans les lipoprotéines séparées par gradient de densité, chez le rat mâle Wistar (a), SW (b) ou RICO (c) ingérant un régime semisynthétique témoin (ST) enrichi ou non en cholestérol (CH) $(0,5 \%)$. a) $\square$, Wistar : moyenne $\pm \mathrm{SEM}, n=8$; 圈 , Wistar $\mathrm{CH}:$ moyenne \pm SEM, $n=8, * p<0,05$ par rapport à Wistar. b) $\square$, SW : moyenne $\pm \mathrm{SEM}, n=6$; 圆, SW CH : moyenne \pm SEM, $n=68,{ }^{*} p<0,05$ par rapport à SW . c) $\square$, RICO : moyenne $\pm \operatorname{SEM}, n=6$; 图, RICO $\mathrm{CH}$ : moyenne \pm SEM, $n=68, * p<0,05$ par rapport à $\mathrm{RICO}$. CM (chylomicrons) + VLDL (lipoprotéines de très basse densité) : $0,95<\mathrm{d}<1,020$. $\mathrm{LDL}=$ lipoprotéines de basse densité : $1,020<\mathrm{d}<$ 1,050. HDL1 = lipoprotéines de basse densité : $1,050<\mathrm{d}$ $<1,069$. HDL2 = lipoprotéines de haute densité : $1,069<\mathrm{d}<1,21$.

sont les LDL $(1,019<\mathrm{d}<1,063)$, produits de l'hydrolyse intraplasmatique des VLDL qui sont essentiellement responsables de l'augmentation de la cholestérolémie totale $[10,15,25]$.

\subsubsection{Autres composés}

La figure 3 illustre l'évolution des différentes fractions de lipoprotéines du plasma chez le rat RICO ingérant pendant $45 \mathrm{j}$ le régime semi-synthétique témoin (sans cholestérol) additionné ou non de thé Tuocha (figure $3 a$ ) ou pendant $90 \mathrm{j}$ un régime témoin enrichi $(0,5 \%)$ en cholestérol et contenant ou non un extrait équivalant de thé Tuocha (figure $3 b$ ). Bien que la cholestérolémie totale de ces animaux soit peu modifiée par l'addition de thé à l'alimentation, on observe une baisse sensible des fractions de très basse densité $(0,95<\mathrm{d}<1,05)$. Lorsque l'alimentation est enrichie en cholestérol, on note les mêmes observations que celles faites plus haut. L'extrait aqueux de thé Tuocha produit, comme précédemment, une baisse sensible des lipoprotéines légères, riches en triglycérides. La figure $3 b$ montre également l'influence d'une ingestion d'allyldisulfide $\left(\mathrm{CH}_{2}=\mathrm{CH}-\mathrm{CH}_{2} \mathrm{~S}-\mathrm{SCH}_{2}-\right.$ 

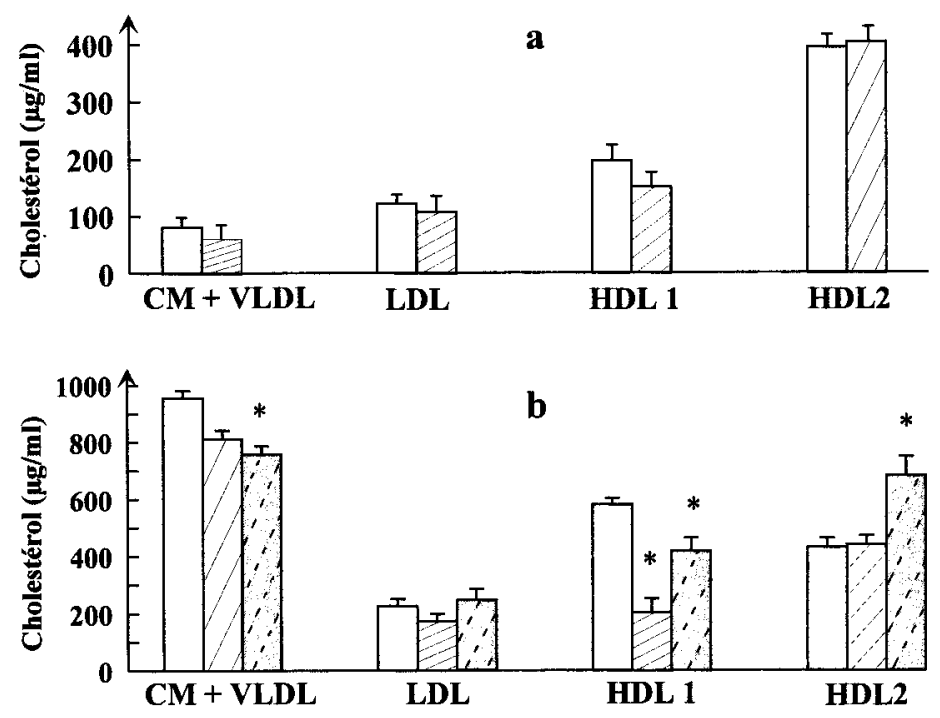

Figure 3. a) Distribution du cholestérol $\left(\mu \mathrm{g} \cdot \mathrm{mL}^{-1}\right.$ de plasma) dans les lipoprotéines séparées par gradient de densité, chez le rat RICO ingérant pendant $45 \mathrm{j}$ une alimentation semi-synthétique témoin sans cholestérol et recevant ou non un extrait sec de thé tuocha (équivalent à $3,75 \%$ ). $\square$, RICO contrôle : moyenne $\pm \mathrm{SEM}, n=6$; $\bigvee Z$, , Thé tuocha : moyenne $\pm \mathrm{SEM}, n=68,{ }^{*} p<0,05$ par rapport à RICO contrôle. b) Distribution du cholestérol $\left(\mu \mathrm{g} \cdot \mathrm{mL}^{-1}\right.$ de plasma) dans les lipoprotéines séparées par gradient de densité, chez le rat RICO ingérant pendant 90 j une alimentation semi-synthétique enrichie en cholestérol $(0,5 \%)$ et recevant ou non du thé $(3,75 \%)$ ou de l'allyldisulfide $(0,15 \%)$. $\square$, RICO : moyenne \pm SEM, $n=6 ; Z 7,+$ Thé : moyenne \pm SEM, $n=68, * p<0,05$ par rapport à $\mathrm{RICO} ; \because$, , allyldisulfide : moyenne $\pm \mathrm{SEM}, n=6,{ }^{*} p<0,05$ par rapport à RICO. CM (chylomicrons) + VLDL (lipoprotéines de très basse densité) : $0,95<\mathrm{d}<1,020$. LDL $=$ lipoprotéines de basse densité $: 1,020<\mathrm{d}<1,050$. $\mathrm{HDL}=$ lipoprotéines de basse densité $: 1,050<\mathrm{d}<1,069$. HDL2 = lipoprotéines de haute densité $: 1,069<\mathrm{d}<1,21$.

$\left.\mathrm{CH}-\mathrm{CH}_{2}\right)$ (extrait d'ail ajouté à raison de $0,15 \%$ dans l'alimentation semi synthétique de base contenant $0,5 \%$ de cholestérol et ingéré pendant 8 semaines) sur la répartition du cholestérol dans les diverses lipoprotéines. Cette concentration d'allyldisulfide ne diminue pas significativement la concentration totale de cholestérol chez les rats $\mathrm{RICO}$, mais elle modifie la distribution du cholestérol dans les différentes lipoprotéines. Les HDL $(1,063<\mathrm{d}<1,21)$ transportent $32,5 \%$ du cholestérol plasmatique au lieu de $20 \%$. L'augmentation absolue et relative du cholestérol dans les HDL " lourdes » est associée à une diminution importante du cholestérol des lipoprotéines riches en triglycérides. Ces observations sont certainement à rapprocher du fait que l'allyldisulfide ou d'autres composés soufrés de l'ail inhibent la biosynthèse hépatique du cholestérol [16-18, 42] et la sécrétion des VLDL par le foie $[16,52]$. Chez l'homme, 0,25 mg d'extrait d'huile lipidique d'ail par kg de poids par jour diminue significativement la cholestérolémie et la triglycéridémie tandis que le cholestérol des HDL s'élève également [4].

\subsection{Effets de diverses drogues sur le métabolisme du cholestérol}

\subsection{1. Éthinylestradiol}

On sait que l'administration d'éthinylestradiol à des rats induit une très importante 
augmentation des LDLr et une baisse très spectaculaire de la cholestérolémie [6, 49]. La figure 4 illustre cet effet chez le rat RICO et l'on note que c'est la fraction HDL1 (riche en apoE) qui est la plus effondrée.

\subsubsection{Statines}

Il était a priori étonnant mais il a été démontré amplement que les statines possèdent très peu d'efficacité pour abaisser la cholestérolémie chez le rat $[11,22,29,48]$. Le rat RICO n'échappe pas à cette règle. Nous avons étudié l'effet de la sinvastatine à la dose de $0,1 \%$ dans l'alimentation pendant un mois sans observer d'effets significatifs sur la cholestérolémie (figure 5).

Notons qu'il en a été de même avec la crilvastatine, une nouvelle molécule de la famille des statines, qui n'a jamais été commercialisée et qui s'est avérée sans efficacité

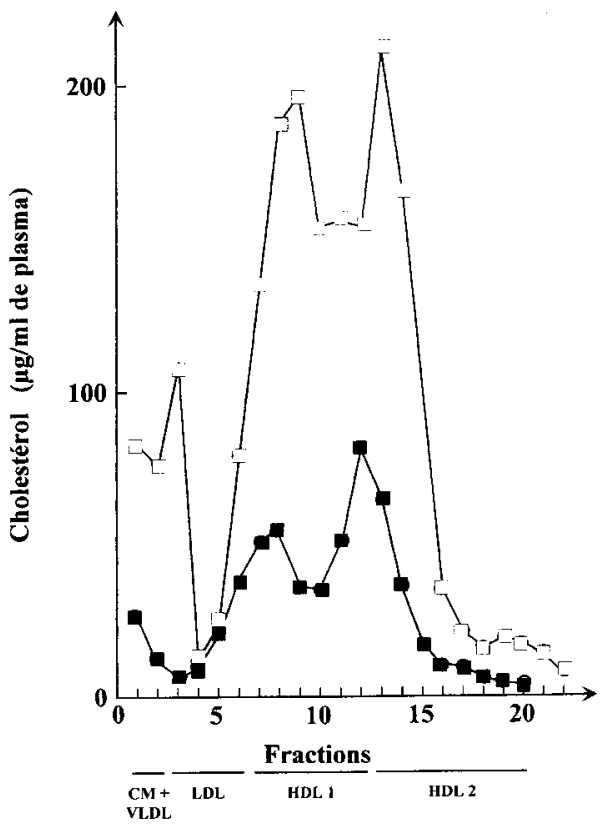

Figure 4. Distribution du cholestérol $\left(\mu \mathrm{g} \cdot \mathrm{mL}^{-1}\right.$ de plasma) dans les lipoprotéines séparées par gradient de densité chez le rat génétiquement hypercholestérolémique RICO témoin ou recevant $5 \mathrm{mg} \cdot \mathrm{kg}^{-1}$ d'éthinylestradiol pendant $5 \mathrm{j}$ (RICO $\mathrm{EE})$. $\square$, RICO témoin ; $\mathbf{Q}, \mathrm{RICO} \mathrm{EE}$. sur la cholestérolémie du rat RICO à la dose journalière de $200 \mathrm{mg} \cdot \mathrm{kg}^{-1}$ alors qu'elle inhibe puissamment la synthèse du cholestérol et l'HMGCoA réductase hépatique [12, 21]. Le manque d'effet de la sinvastatine ou de la crilvastatine sur la cholestérolémie est certainement la conséquence du fait que, contrairement à l'homme, on n'observe pas d'augmentation du nombre des LDLr sous l'effet de ces deux statines chez le rat (observations non publiées de l'auteur). En conséquence, il n'y a pas stimulation de l'épuration plasmatique des LDL et des HDL1 (ou HDL riches en apoE), lipoprotéines très efficacement épurées par ce récepteur [45].

\subsubsection{F 2833}

A contrario de l'exemple précédent, un autre composé que nous avons étudié (le F2833), qui n'a également pas été commercialisé, s'est avéré être un puissant stimulateur des LDLr chez le rat RICO, sans qu'il modifie significativement l'absorption intestinale du cholestérol ou la synthèse hépatique du cholestérol. À la dose de $150 \mathrm{mg} \mathrm{kg} \cdot \mathrm{j}^{-1}$, ce composé stimulait très efficacement l'épuration des «LDL » plasmatiques (lipoprotéines de densité comprise entre 1020 et $1050)$ du rat RICO et apparaissait donc comme un hypocholestérolémiant très efficace de cette fraction, chez cet animal (figure 6).

\subsubsection{Influence de la streptozotocine et d'un inhibiteur de l'ACAT}

Un dernier exemple intéressant concerne l'effet d'un inhibiteur de l'acylCoA-cholestérol acyl transférase (ACAT) sur les lipoprotéines du rat RICO rendu diabétique par une injection unique de streptozotocine (50 $\mathrm{mg} \cdot \mathrm{kg}^{-1}$ ) (figure 7 ). Dès $6 \mathrm{j}$ (résultats non montrés) ou $28 \mathrm{j}$ (figure $7 a$ ) après l'administration de la streptozotocine, les lipoprotéines du rat RICO sont profondément modifiées : il y a une augmentation importante des HDL « lourdes » ( HDL2 de densité 1,063 à 1,21 ) et une diminution des 
Figure 5. Distribution du cholestérol $\left(\mu \mathrm{g} \cdot \mathrm{mL}^{-1}\right.$ de plasma) dans les lipoprotéines séparées par gradient de densité, chez le rat RICO ingérant pendant un mois une alimentation semi-synthétique témoin avec ou sans $0,1 \%$ de sinvastatine. $\square$, RICO : moyenne \pm SEM, $n=6$ par groupe $; \bullet$, RICO $+\mathrm{O}, 1 \%$ de sinvastatine.

Figure 6. Distribution du cholestérol $\left(\mu \mathrm{g} \cdot \mathrm{mL}^{-1} \mathrm{de}\right.$ plasma) dans les lipoprotéines séparées par gradient de densité, chez le rat RICO ingérant pendant $90 \mathrm{j}$ une alimentation semi-synthétique témoin avec ou sans $150 \mathrm{mg} \cdot \mathrm{kg}^{-1} \cdot \mathrm{j}^{-1}$ de F 2833. $\square$, RICO contrôle : moyenne \pm SEM, $n=6$ par groupe ; 图, F2833 (3 mois de traitement, $\left.150 \mathrm{mg} \cdot \mathrm{kg}^{-1} \cdot \mathrm{j}^{-1}\right)$ : moyenne \pm SEM, $n=6$ par groupe, $* * * p<0,001$ par rapport à RICO contrôle.
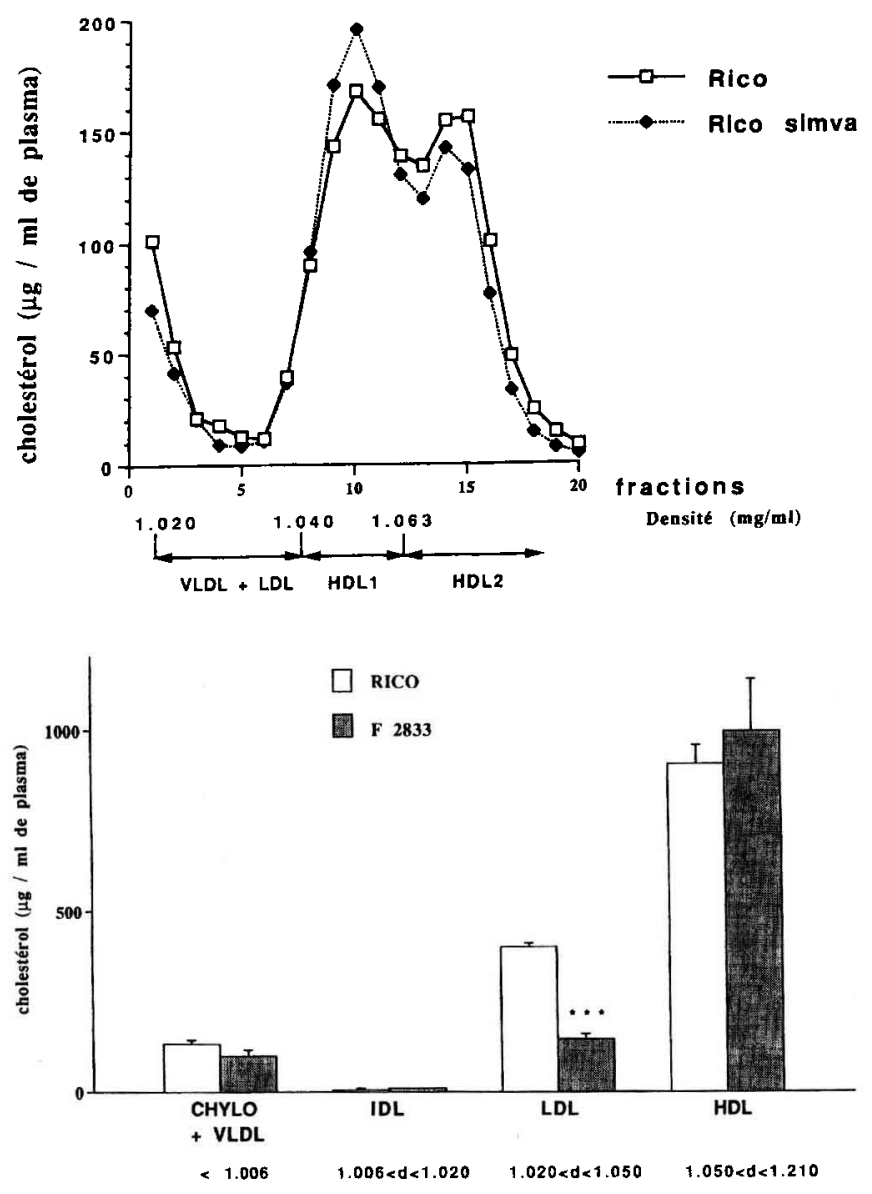

HDL « légères », riches en apoE (HDL1, de densité 1,040 à 1,063 ), alors même que la cholestérolémie totale est assez peu modifiée. Ces modifications vont de pair avec une augmentation spectaculaire du coefficient d'absorption du cholestérol alimentaire qui passe de 28 à $47 \%$ chez le diabétique (figure $7 b$ ) [34]. Il était donc intéressant de tester un inhibiteur de l'ACAT qui inhibe l'absorption intestinale du cholestérol afin d'examiner si, chez le rat RICO diabétique, le spectre lipoprotéinique allait être amélioré. La figure $7 a, b$ montre qu'il en est bien ainsi. On observe, chez les rats RICO diabétiques recevant l'inhibiteur de l'ACAT, un retour à la normale du cœfficient d'absorption intestinal du cholestérol et un quasi-retour à la normale du spectre des lipoprotéines du plasma. Nous avons observé, de plus, que cette modification du spectre des lipoprotéines chez le rat RICO diabétique (abaissement des HDL « légères » et augmentation des HDL « lourdes ») est parallèle à une augmentation des LDL récepteurs et à une baisse des $\mathrm{HB}_{2}$ (protéines de liaison aux HDL, mises en évidence et clonées par Matsumoto et al. [32]). Ces observations confirment le rôle important des LDLr dans l'épuration des HDL riches en apoE et suggèrent, de plus, un rôle possible de ces dernières protéines de liaisons aux $\mathrm{HDL}\left(\mathrm{HB}_{2}\right)$ dans l'épuration des HDL lourdes [33]. 

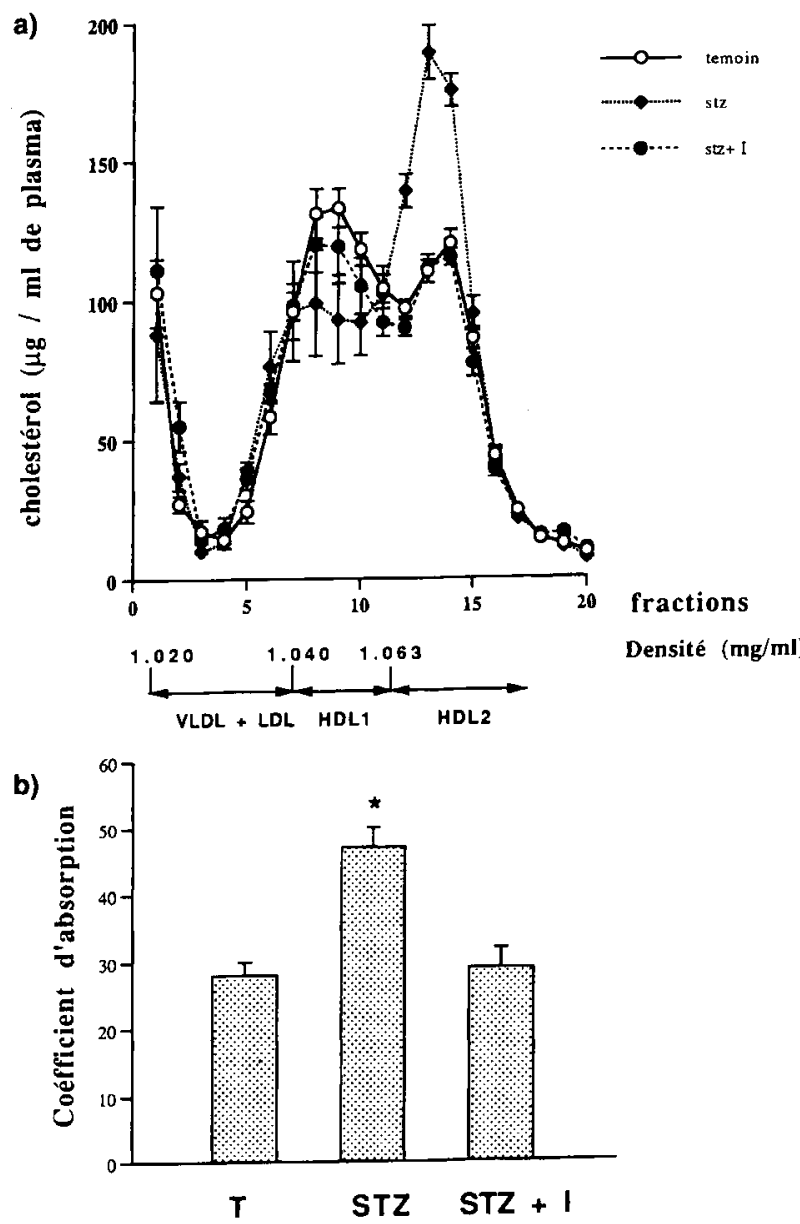

Figure 7. a) Distribution du cholestérol $\left(\mu \mathrm{g} \cdot \mathrm{mL}^{-1} \mathrm{de}\right.$ plasma) dans les lipoprotéines séparées par gradient de densité, chez le rat RICO normal, rendu diabétique par une injection unique $\left(50 \mathrm{mg} \cdot \mathrm{kg}^{-1}\right.$ ) de streptozotocine et diabétique et recevant $20 \mathrm{mg} \cdot \mathrm{kg}^{-1} \cdot \mathrm{j}^{-1}$ pendant $28 \mathrm{j}$ un inhibiteur de I'ACAT (I). O, RICO : moyenne $\pm \mathrm{SEM}, n=7$; STZ: moyenne \pm SEM, $n=5$, $* p<0,05$ par rapport à RICO ; , STZ + I : moyenne $\pm \mathrm{SEM}, n=7$. b) Coefficient d'absorption du cholestérol alimentaire, mesuré par le principe d'occupation, chez les rats RICO, STZ, et STZ + I sacrifiés après $28 \mathrm{j}$ d'expérience $(* p<0,05$ par rapport à RICO).

Le rat (RICO ou non) et l'homme possédant un spectre lipoprotéinique différent, il est légitime de se demander si les variations d'une population de lipoprotéines plasmatiques chez le rat RICO peuvent correspondre à celles d'une population de lipoprotéines plasmatiques, chez l'homme. De plus, ces variations peuvent-elles être corrélées aux modulations d'un (ou de plusieurs) processus importants du métabolisme du cholestérol ?

Chez le rat, dès 1976, il a été mis en évidence [8] une relation inverse entre la concentration du cholestérol total du plasma et la sécrétion interne de cholestérol (cho- lestérogenèse de l'ensemble des tissus de l'organisme qui se déverse dans le plasma), pour un débit donné de cholestérol absorbé (figure 8a). Par ailleurs, la cholestérolémie du rat est corrélée à son temps de renouvellement dans le plasma: en d'autres termes, elle augmente d'autant plus que son taux de renouvellement (FCR) s'abaisse ou que son temps de renouvellement s'accroît (figure $8 b$ ). De même, une relation inverse a été observée entre le débit de la cholestérogenèse et l'absorption intestinale de cholestérol (figure 8c) [7].

Chez l'homme, une même relation entre cholestérolémie totale et temps de renou- 

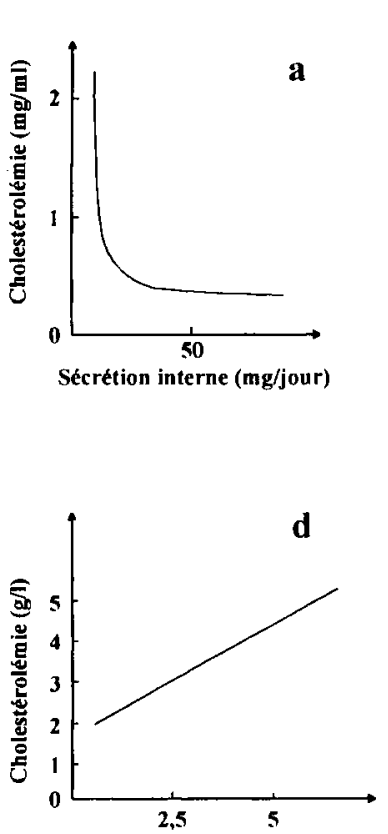

T. de renouvellement ou $1 / \mathrm{FRC}$ (jour//)

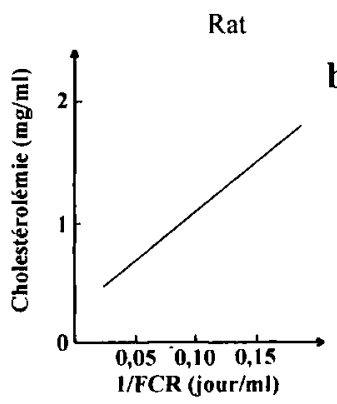

b

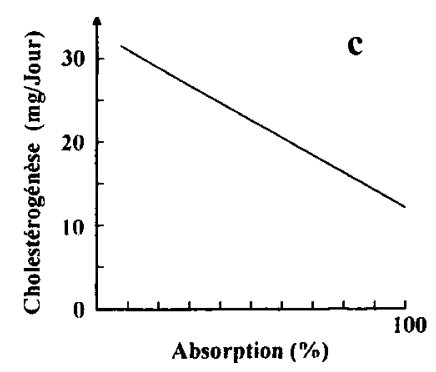

Hornme
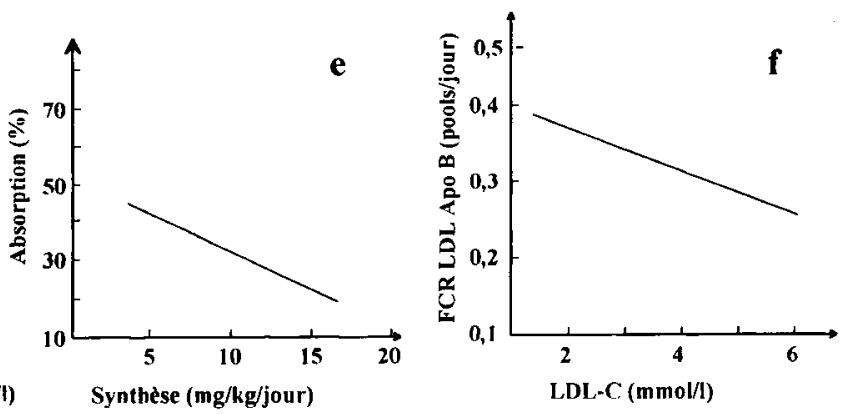

Figure 8. Rat - a) Concentration du cholestérol plasmatique en fonction du débit de secrétion interne (synthèse), chez le rat [8]. b) Concentration du cholestérol plasmatique en fonction du temps de renouvellement (inverse du taux de renouvellement ou FCR) chez le rat [8]. c) Valeurs $\left(\mathrm{mg} \cdot \mathrm{j}^{-1}\right.$ ) des vitesses de synthèse du cholestérol en fonction du coefficient d'absorption intestinale du cholestérol chez des rats soumis à 30 conditions expérimentales différentes [7]. Homme - d) Concentration du cholestérol plasmatique en fonction du temps de renouvellement (inverse du taux de renouvellement ou FCR), chez l'homme [9]. e) Absorption intestinale du cholestérol chez l'homme en fonction de la synthèse [19]. f) Taux de catabolisme (FCR, en pool $\left.{ }^{-1}\right)$ en fonction de la concentration du LDL cholestérol, chez l'homme) [20].

vellement a été mise en évidence par Chevallier et al. [9] (figure 8d) et, plus récemment, par Gylling et al. [20] ; Gylling et Miettinen [19], une corrélation entre l'absorption et la synthèse de cholestérol (figure $8 e$ ) ou entre le FCR des LDL et la concentration plasmatique du LDLcholestérol (figure 8f). Ainsi, les variations de la cholestérolémie reflètent essentiellement celles de la "LDLémie ", chez l'homme, et celles de la « HDLémie », chez le rat.

Plus récemment, partant de l'analyse des valeurs individuelles de trois groupes de rats ingérant ou non un régime enrichi en cho- lestérol ou en cystine ( $n=12)$, une corrélation significative a été obtenue entre le cholestérol plasmatique porté par la fraction HDL1 $(1,040<\mathrm{d}<1,063)$ et la synthèse hépatique du cholestérol [43]. L'analyse des lipoprotéines du plasma et l'estimation de la synthèse de cholestérol après incorporation d'acétate ${ }^{14} \mathrm{C}$ dans les stérols hépatiques a donc été faite chez huit groupes de rats (Wistar, SW ou RICO) ingérant soit un régime semi-synthétique à base de saccharose, ou ce régime supplémenté en cystine ou en cholestérol, composés qui modifient beaucoup la synthèse hépatique du cholestérol. La figure 9 montre qu' une corrélation 


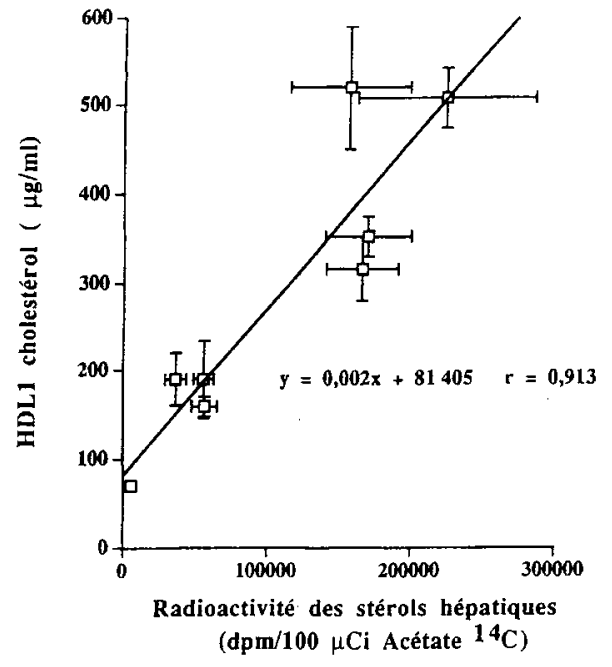

Figure 9. Concentration du cholestérol des HDL1 plasmatiques en fonction de la radioactivité des stérols hépatiques (dpm), 70 min après injection d'acétate $-{ }^{14} \mathrm{C}(3.7 \mathrm{Mbq})$ chez des rats Wistar, SW ou RICO ingérant un régime semi-synthétique témoin, enrichi ou non en cystine $(5 \%)$ ou en cholestérol $(1 \%): y=0,002 x+81405$, $\mathrm{r}=0,913 ;{ }^{*} p<0,002$ (d'après Serougne et al. [43] ; Cardona-Sanclemente et al. [5] ; Hajri et al. [21]).

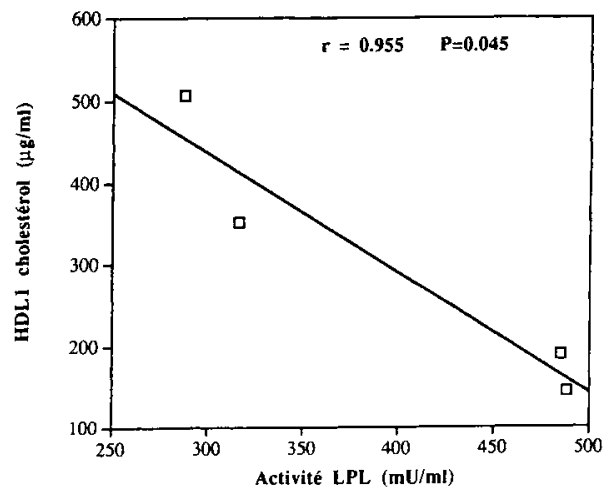

Figure 10. Concentration en cholestérol des HDLl du plasma en fonction de l'activité lipoprotéine lipase (LPL) relarguable par l'héparine dans le plasma chez des rats SW et RICO ingérant ou non $0,5 \%$ de cholestérol dans leur alimentation semi-synthétique témoin : $y=-1,466 x$ $+875,969 ; r=0,955,{ }^{*} p<0,05$ (d'après Cardona-Sanclemente et al. [5] ; Sultan et al. [47]). significative est également obtenue à partir des valeurs moyennes de ces huit lots de rats de souches différentes $(n=48$; $y=0,002 x+81405 ; r=0,913)$. Ceci montre l'importance de la synthèse de cholestérol par le foie du rat dans l'origine et la sécrétion des HDL1. Nous avons d'ailleurs récemment observé que, $4 \mathrm{j}$ après une irradiation $\gamma$ modérée (4 Gy), il y a hypersynthèse de cholestérol dans le foie du rat et augmentation spectaculaire de la fraction HDL1, cette dernière retournant à la normale $12 \mathrm{j}$ après l'irradiation, de même que la cholestérogenèse hépatique [14].

Chez des rats RICO ou SW ingérant ou non du cholestérol $(0,5 \%)$ dans leur alimentation semi-synthétique témoin, la mesure des activités LPL et HL relarguables par l'héparine a été effectuée parallèlement à l'analyse des lipoprotéines du plasma [47]. Une relation inverse significative est observée entre la concentration de cholestérol porté par les HDL1 $(y=-1,466 x+875,97$, $\mathrm{r}=0,955, n=24, p<0.045)$ et l'activité LPL (figure 10). De même, nous observons souvent une proportionnalité entre la concentration de cholestérol des HDL et l'activité LPL ou une relation inverse entre le cholestérol porté par les HDL et l'activité de la lipase hépatique (HL) (résultats non montrés). Ces résultats soulignent, une fois encore, l'importance du rôle des enzymes lipolytiques dans la formation de ces lipoprotéines : une corrélation entre HDLl et activité HL avait déjà été observée chez le rat [30]. Dans cette espèce, les HDL3, produites à partir des matériaux obtenus au cours de l'hydrolyse des lipoprotéines riches en triglycérides sont transformées en HDL2 par 1'action de la LCAT, puis en HDL1, du fait de l'absence (ou de l'inhibition) de la CETP [51]. Les HDL1 du rat, produits finaux de l'hydrolyse intraplasmatique peuvent ainsi être rapprochées des LDL humaines, produits de l'hydrolyse intraplasmatique des VLDL. On a observé plus haut que la fraction HDL 1 est très abaissée chez le rat RICO rendu diabétique par une injection de streptozotocine (figure 7). 
Dans ces conditions, on sait aussi que l'activité de la LPL s'avère significativement augmentée [46].

L'ensemble de ces travaux montrent, enfin, qu'un composé hypocholestérolémiant chez une espèce animale (le rat, par exemple) peut être inefficace chez l'homme (et vice-versa). L'exemple de la sinvastatine qui stimule fortement les LDLr humains et non les murins est tout à fait instructif, à cet égard. Il apparaît cependant clair que tout composé qui stimule les LDLr du rat RICO et de l'homme induit une baisse des HDL (à apoE) chez le rat et une baisse des LDL chez l'homme. On voit donc, à nouveau, l'importance qu'il y a de stimuler le catabolisme des LDL chez l'homme (et des HDL chez le rat) pour abaisser la cholestérolémie. Généralement, la cholestérogenèse hépatique augmente sensiblement, en retour, mais la cholestérolémie, reflétée principalement par la LDLémie chez l'homme, est abaissée. On note également qu'une inhibition spécifique de la synthèse hépatique du cholestérol par une drogue n'est donc pas une fin en soi : l'action hypocholestérolémiante de la sinvastatine n'est pas due à son inhibition de l'HMGCoAR mais est surtout la conséquence de son effet de stimulation importante des LDLr, chez l'homme. De plus, cette drogue abaisse l'absorption intestinale du cholestérol [23]. Chez le rat, où elle est inefficace pour stimuler le nombre de LDLr, son effet hypocholestérolémiant est insignifiant ou nul.

En conclusion, le rat génétiquement hypercholestérolémique RICO - dont la cholestérolémie approche celle de l'humain apparaît comme un modèle intéressant pour tester l'action d'un composé de l'alimentation ou une drogue susceptible d'agir sur une étape-clé du métabolisme du cholestérol. Bien que son spectre de lipoprotéines plasmatiques soit celui d'un murin (HDL dominantes), une large part du cholestérol plasmatique est portée par des HDL1 (HDL à apoE), épurées par les LDLr, lipoprotéines que l'on peut comparer aux LDL, princi- pale classe de transporteurs du cholestérol dans le plasma de l'homme. Ainsi, les statines qui ne modifient pas le nombre de LDLr chez le rat (RICO ou non) contrairement à ce qui est observé chez l'humain, sont sans effet sur la cholestérolémie (et les HDL1) du rat RICO alors que l'estradiol, la streptozotocine ou d'autres drogues (F2833...) qui stimulent fortement la quantité de ces récepteurs possèdent un effet " hypoHDL1 » et hypocholestérolémiant puissant.

\section{REMERCIEMENTS}

L'auteur tient à remercier plus particulièrement ses principaux collaborateurs impliqués dans les études du métabolisme du cholestérol chez le rat RICO (Mesdames Marie-France Blouquit, Jacqueline Férézou, Colette Sérougne, Messieurs Daniel Gripois, Thierry Magot, Michel Parquet, Michel Riottot) ainsi que les jeunes chercheurs qui ont participé activement à la vie scientifique du laboratoire au cours de ces dix dernières années (L.E Cardona-Sanclemente, J. Khallou, T. Hajri, K. Ouguerram ). La précieuse assistance technique de Madame Claudine Verneau et de Monsieur Fabien Milliat pour le dépouillement chimique des expériences, et pour la réalisation des illustrations est vivement appréciée.

Grand merci également pour les collaborations et les discussions très précieuses, fructueuses et amicales de Sabine Griglio, Denis Mathé, André Mazur et Fabrice Sultan.

\section{RÉFÉRENCES}

[1] Barbara L., Sama S., Morsell-Labate A.M., Taroni F., Rusticalli A.G., Festi D., Sapio C., Roda E., A population study on the prevalence of gallstone disease: The sirmione study, Hepatology 7 (1987) 913-917.

[2] Bartram H.P., Scheppach W., Heid C., Fabian C., Kasper H., Effect of starch malabsorption on fecal bile acids and neutral sterols in Humans: possible implications for colonic carcinogenesis, Cancer Res. 51 (1991) 4238-4242.

[3] Behall K.M., Howe J.C., Effect of long-term consumption of amylose vs amylopectin starch on metabolic variables in human subject, Am. J. Clin. Nutr. 61 (1995) 334-340. 
[4] Bordia A., Effect of garlic on blood lipids in patients with coronary heart disease, Am. J. Clin. Nutr. 34 (1981) 2100-2103.

[5] Cardona-Sanclemente L.E., Ferezou J., Lutton C., Cholesterol metabolism in the genetically hypercholesterolemic (RICO) rat. II A study of plasma lipoproteins and effect of dietary cholesterol, Biochim. Biophys. Acta 960 (1988) 382-389.

[6] Chao Y., Windler E.E., Chen G.C., Havel R.J., Hepatic catabolism of rat and human lipoproteins in rats treated with $17 \alpha$-Ethinyl Estradiol, J. Biol. Chem. 254 (1979) 11360-11366.

[7] Chevallier F., Lutton C., The intestine is the major site of cholesterol synthesis in the rat, Nat. New Biol. 242 (1973) 61-62.

[8] Chevallier F., Mathe D., Lutton C., Lois des taux de cholestérol dans le plasma et le foie de rats adultes, C. R. Acad. Sci. Paris 283 (1976) 1759-1762.

[9] Chevallier F., Ferezou J., Rautureau J., A new view of human cholesterolemia, Path. Biol. 28 (1979) 173-179.

[10] Dubois C., Armand M., Mekki N., Portugal H., Pauli A.M., Bernard P.M., Lafont H., Lairon D., Effects of increasing amounts of dietary cholesterol on postprandial lipemia and lipoproteins in human subjects, J. Lipid Res. 35 (1994) 1993-2007.

[11] Endo A., Tsujita Y., Kuroda M., Tanzawa K., Effects of ML-236B on cholesterol metabolism in mice and rats: lack of hypocholesterolemic activity in normal animals, Biochim. Biophys. Acta 575 (1979) 266-276.

[12] Esnault C., Lafont H., Chanussot F., Chauton M., Hauton J., Laruelle C., Inhibition of hepatic HMGCoA reductase activity by two new hypocholesterolemic drugs, in: Guillouzou A. (éd.), Liver Cells and Drugs, Colloque Inserm 164, Inserm, Paris, 1988, p. 99.

[13] Felgines C., Sérougne C., Mazur A., Férézou J., Lutton C., Apolipoprotein synthesis and LDLreceptor gene expression in the genetically hypercholesterolemic rat (Rico), Atherosclerosis 117 (1995) 15-24.

[14] Feurgard C., Bayle D., Guezingar F., Serougne C., Mazur A., Lutton C., Aigueperse J., Gourmelon P., Mathe D., Effects of ionizing radiation (neutron/gamma) on rat plasma lipids and lipoproteins, Radiat. Res. 150 (1998) 43-51.

[15] Fless G.M., Scanu A.M., Comparative study of density distribution of plasma lipoproteins of normo-and hypercholesterolemic rhesus monkeys and humans, Arteriosclerosis 6 (1986) 88-97.

[16] Gebhart R., Inhibition of cholesterol biosynthesis by a water-soluble garlic extract in primary cultures of rat hepatocytes, Arz. forsch. 41 (1991) 800-804.
[17] Gebhardt R., Amplification of palmitate-induced inhibition of cholesterol biosynthesis in cultured rat hepatocytes by garlic-derived organosulfur compounds, Phytomedicine 2 (1996) 29-34.

[18] Gebhardt R., Beck H., Differential inhibitory effects of garlic-derived organosulfur compounds on cholesterol biosynthesis in primary rat hepatocyte cultures, Lipids 31 (1996) 1269-1276.

[19] Gylling H., Miettinen T.A., The effect of cholesterol absorption inhibition on low density lipoprotein cholesterol level, Atherosclerosis 117 (1995) 305-308.

[20] Gylling H., Strandberg T., Tilvis R., Miettinen T.A., Regulation of serum cholesterol level in middle-aged and elderly Men, Arterioscler. Thromb. 14 (1994) 694-700.

[21] Hajri T., Férézou J., Laruelle C., Lutton C., Crilvastatin, a new HMG-CoAR inhibitor, inhibits cholesterol absorption in the genetically hypercholesterolemic rats, Eur. J. Pharmacol. 286 (1995) 131-136.

[22] Hirano T., Kumuro F., Furukawa S., Nagano S., Takahashi T., Effect of pravastatin sodium, e new inhibitor of 3-hydroxy-3-methyl glutarylCoA reductase on very low density lipoprotein composition and kinetics in hyperlipidemia associated with experimental N, Metabolism 39 (1990) 605-609.

[23] Hunninghake D.B., HMGCoA reductase inhibitors, Curr. Opin. Lipidol. 3 (1992) 22-28.

[24] Khallou J., Riottot M., Verneau C., Parquet M., Lutton C., Antilithiasic and hypocholesterolemic effects of diets containing autoclaved amylomaize starch in lithiasic hamster, Dig. Dis. Sci. 40 (1995) 2540-2549.

[25] Kieft K.A., Bocan T.M.A., Krause B.R., Rapid on-line determination of cholesterol distribution among plasma lipoproteins after high-performance gel filtration chromatography, J. Lipid Res. 32 (1991) 859-866.

[26] Lutton C., Comparison of isotopic equilibrium and dual isotope blood ratio method for the measurement of cholesterol absorption in rats, Digestion 20 (1980) 346-350.

[27] Lutton C., Anatomical heterogeneity of the intestinal mucosa and cholesterol homeostasis: A review of studies with normal and genetically hypercholesterolemic (RICO) Rat, Digestion 57 (1996) 1-10.

[28] Lutton C., Ferezou J., Serougne C., Verneau C., Champarnaud G., Magot T., Mathe D., Sulpice JC., Critical analysis of the use of $14 \mathrm{C}$ acetate for measuring in vivo rat cholesterol synthesis, Reprod. Nutr. Dev. 30 (1990) 71-84.

[29] Maeda E., Yoshino G., Matsushita M., Nagata K., Morita M., Murata Y.,Naka Y., Kazumi T., Effect of a 3-hydroxy-3-methylglutaryl CoA reductase inhibitor on triglyceride kinetics in chronically streptozotocin-diabetic rats, Metabolism 42 (1993) 52-57. 
[30] Mathé D., Serougne C., Ferezou J., Lecuyer B., Lipolytic activities in rats fed a sucrose-diet supplemented with either cystine or cholesterol: relationship with lipoprotein profiles, Ann. Nutr. Metab. 35 (1991) 165-173.

[31] Mathe D., Riottot M., Rostaqui N., Sacquet E., Navarro N., Lecuyer B., Lutton C., Effect of amylomaize starch on plasma lipoproteins of lean and obese Zucker rats, J. Clin. Biochem. Nutr. 14 (1993) 1-8.

[32] Matsumoto A., Mitchell A., Kurata H., Pyle Y., Kondo K., Itakura H., Fidge N., Cloning and characterization of HB2, a candidate high density lipoprotein receptor, J. Biol. Chem. 272 (1997) 16778-16782.

[33] Milliat F., Serougne C., Gripois D., Lutton C., Effects of insulin deficiency on lipoproteins and their hepatic receptors in RICO rats, C. R. Acad. 321 (1998) 999-1006.

[34] Milliat F., Gripois D., Blouquit M.F., Ferezou J., Serougne C., Lutton C., Effects of stretozotocininduced diabetes on the intestinal absorption of dietary cholesterol, plasma lipoproteins and liver lipoprotein receptors (LDLr, SRB1, HB2) in genetically hypercholesterolemic RICO rats, (1999) soumis. .

[35] Morgan L.M., The effect of non-starch polysaccharide supplementation on circulating bile acids, hormone and metabolite levels following a fat meal in human subjects, Br. J. Nutr. 70 (1993) 491-501.

[36] Mortimer B.C., Beveridge D.J., Phan C.T., Lutton C., Redgrave T.G., The diurnal rythms of cholesterol metabolism and plasma clearance of model chylomicrons: comparison of normal and genetically hypercholesterolemic rats (RICO), Comp. Biochem. Physiol. Part A 120 (1998) 671-680.

[37] Muller K.R., Li J.R., Dinh D.M., Subbiah M.T.R., The characteristics and metabolism in the genetically hypercholesterolemic rat, Biochim. Biophys. Acta 574 (1979) 334-343.

[38] Ouguerram K., Magot T., Lutton C., Alterations in cholesterol metabolism in the genetically hypercholesterolemic RICO rat: an overview, 257-274, in: Malmendier C.L., Alaupovic P., Brewer H.B. (éds.), Hypercholesterolemia, Hypocholesterolemia, Hypertriglyceridemia, in vivo Kinetics, Advances in Experimental Medicine and Biology, Plenum Press, New York, 1991, p. 285.

[39] Ouguerram K., Magot T., Lutton C., Metabolism of plasma lipoproteins in the genetically hypercholesterolemic Rat (RICO), Metabolism 45 (1996) 4-11.

[40] Riottot M., Lutton C., Dietary fiber and modulation of bile acid synthesis, in : Lairon D. (éd.), COST 92, Mechanisms of action of fibre on lipid and cholesterol metabolism (Proceedings),
Commission of the European Communities, 1994, pp. 77-84.

[41] Roach P.D., Balasubramaniam S., Hirata F., Abbey M., Szanto A., Simons L.A., Nestel P.J., The low density lipoprotein receptor and cholesterol synthesis are affected differently by dietary cholesterol in the rat, Biochim. Biophys. Acta 1170 (1993) 165-172.

[42] Sendl A., Schliack M., Loser R., Stanislaus F., Wagner H., Inhibition of cholesterol synthesis in vitro by extracts and isolated compounds prepared from garlic and wild garlic, Atherosclerosis 94 (1992) 79-85.

[43] Serougne C., Ferezou J., Rukaj A., A new relationship between cholesterolemia and cholesterol synthesis determined in rats fed an excess of cystine, Biochim. Biophys. Acta 921 (1987) 522-530.

[44] Serougne C., Mathé D., Férézou J., Bertin C., Riottot M., Lutton C., Jacotot B., Effects of simvastatin on plasma lipoprotein concentrations and Very Low Density Lipoprotein secretion in the Rat, J. Clin. Biochem. Nutr. 18 (1995) 55-64.

[45] Serougne C., Feurgard C., Hajri T., Champarnaud G., Ferezou J., Mathe D., Lutton C., Catabolism of HDLl cholesteryl ester in the Rat. Effect of ethinyl estradiol treatment, C. R. Acad. 322 (1999) 1-6.

[46] Sheperd G., Cam M.C., Sambandam N., Abrahani M.A., Rodrigues B., Streptozotocin-induced diabetes enhances cardiac-releasable lipoprotein lipase activity in spontaneously hypertensive rats, Hypertension 31 (1998) 878-884.

[47] Sultan F., Cardona-Sanclemente L.E., Lagrange D., Lutton C., Griglio S., Lipoprotein lipase and hepatic activities in a hypercholesterolemic (RICO) strain of Rat. Effect of dietary cholesterol, Biochem. J. 266 (1990) 340-353.

[48] Van Tol A., Jansen E.H.J.M., Koomans H.A., Joyles J.A.,. Hyperlipoproteinemia in Nagase analbuminemic rats: effects of pravastatin on plasma (apo)lipoproteins and lecithin:cholesterol acyltransferase activity, J. Lipid Res. 32 (1991) 1719-1728.

[49] Windler E.E.T., Kovanen P.T., Chao Y., Brown M.S., Havel R.J., Goldstein J.L., The Estradiol-stimulated lipoprotein receptor of rat liver, J. Biol. Chem. 255 (1980) 10464-10471.

[50] Wursch P., Starch in human nutrition, Nutr. Value Cereal. Products 60 (1989) 199-256.

[51] Ya Y.C., Barter PJ., Differences in plasma cholesteryl ester transfer activity in sixteen vertebrate species, Comp. Biochem. Physiol. 71B (1982) 265-269.

[52] Yeh Y.Y., Yeh S.M., Garlic reduces plasma lipids by inhibiting hepatic cholesterol and triacylglycerol synthesis, Lipids 29 (1994) 189-193. 